\title{
Discovering the relationship between dietary nutrients and cortisol and ghrelin hormones in horses exhibiting oral stereotypic behaviour: a review
}

\begin{abstract}
This review focuses on associations of cortisol and the hormone ghrelin on abnormal oral behaviors, predominantly stereotypic behavior, in horses. Abnormal oral behaviors are prevalent in the stabled horse population. Feeding practice and satiety seem to play a significant role in the development of the behaviors. The effect of macronutrients on the occurrence of abnormal oral behaviors and satiety remains to be elucidated in horses. Ghrelin, known as the hunger hormone, is produced by the ghrelinergic cells in the gastrointestinal tract. Ghrelin is found to be involved in various physiological processes such as stress, eating disorders, and food selection. Feed preference in horses is chiefly controlled by their ability to distinguish macronutrients in the food, so the novel approach of the use of ghrelin to tackle the problem in the abnormal oral behaviors may provide promising findings in future research of the occurrence and management of equine stereotypies.
\end{abstract}

Keyword: Abnormal behavior; Oral stereotypy; Redirected; Macronutrient; Cortisol; Ghrelinequine 